\title{
Facturación y cuentas médicas en el sector público: Información contable en un hospital público
}

\author{
Edgardo Serafín Passos Simancas \\ Universidad Simón Bolívar
}

Colombia

Francisco José Arias Aragonés Institución Tecnológica Colegio Mayor de Bolívar

Colombia

Armando Batista Castillo Institución Tecnológica Colegio Mayor de Bolívar Colombia

Wilson Guacarí Villalba Institución Tecnológica Colegio Mayor de Bolívar

Colombia

Adriana María Flórez Laiseca

Institución Tecnológica Colegio Mayor de Bolívar

Colombia 


\title{
Facturación y cuentas médicas en el sector público: Información contable en un hospital público
}

\author{
Edgardo Serafín Passos Simancas ${ }^{1,2}\left(\mathbb{D}\right.$, Francisco José Arias Aragonés ${ }^{2} \mathbb{D}^{\circ}$, Armando \\ Batista Castillo $^{2}$ iD y Wilson Guacarí Villalba $^{2}$ iD \\ ${ }^{1}$ Universidad Simón Bolívar, Colombia \\ ${ }^{2}$ Institución Tecnológica Colegio Mayor de Bolívar, Colombia
}

Para citaciones: Passos, E., Arias, F., Batista, A. y Guacarí, W. (2020). Facturación y cuentas médicas en el sector público: Información contable en un hospital público. Panorama Económico, 28(1), 41-56. https://doi.org/10.32997/pe-2020-2669

Recibido: 23/05/2019

Aprobado: 17/08/2019

Autor de correspondencia: Edgardo Serafín Passos Simancas epassos1@unisimonbolivar.edu.co

Editor: Andrés Escobar Espinoza Universidad de Cartagena, Colombia.

Tipología IBN Publindex: Artículo de investigación científica y tecnológica.

Copyright: (C) 2020. Passos, E., Arias, F., Batista, A. y Guacarí, W. Este es un artículo de acceso abierto, distribuido bajo los términos de la licencia https://creativecommons.org/licenses/bync-sa/4.0/ la cual permite el uso sin restricciones, distribución y reproducción en cualquier medio, siempre y cuando que el original, el autor y la fuente sean acreditados.

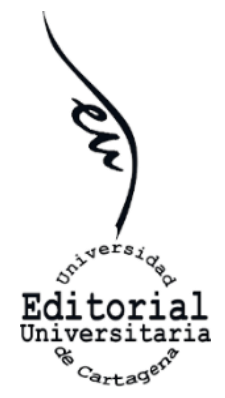

\section{RESUMEN}

El presente documento analiza los procesos de facturación y cuentas médicas de la Empresa Social del Estado (ESE) Hospital Local de Arjona, Colombia, mediante la aplicación de instrumentos de captura de información al personal del área de contabilidad. Se utiliza el diagnóstico organizacional como herramienta de evaluación de la gestión de estos dos procesos, considerados críticos en las organizaciones dedicadas a la prestación de servicios de salud. La problemática estriba en que con mucha frecuencia se presentan objeciones o "glosas", atribuibles a inconsistencias e irregularidades en los procesos de facturación y presentación de las cuentas médicas. Lo anterior conduce a que no se haga el pago de la cuenta y con ello se desencadena un problema que amenaza la sostenibilidad y la calidad de la prestación de los servicios de salud en las organizaciones dedicadas a este sector. Se encontró que la presencia de inconsistencias y deficiencias en el proceso de facturación y cuentas médicas son atribuibles a la ausencia de control en la facturación y debilidades en los procesos de radicación, auditoría y respuesta oportuna de glosas. Se concluye que el problema de sostenibilidad financiera que exhibe la institución es el mismo que ha ahondado la crisis que padece el sector a nivel nacional asociado con el desconocimiento de la normativa para la presentación y estructuración de las cuentas médicas y unificación de criterios en estos procesos.

Palabras clave: Facturación; cuentas médicas; glosa; sostenibilidad; hospital. JEL: M40, M41, M42

\section{Medical bills and billing: cost information in a state-owned hospital}

\section{ABSTRACT}

This document analyzes the billing and medical bills processes of the state-owned hospital of Arjona, Colombia. Organizational assessment is used as a tool for evaluating these two processes, both considered as critical points in organizations dedicated to the provision of health services, in particular state-owned hospitals. The problem is that objections are frequently presented, as outcomes of inconsistencies and irregularities in billing practices and medical bill presentation. This leads to the non-payment of bills, and consequently emerges a serious problem that threatens the sustainability and quality of the provision of health services. It was found that the presence of inconsistencies and deficiencies are consequences of the lack of control in the billing process and to the weaknesses in the processes of filing, auditing and timely response. It is concluded that the problem of financial sustainability exhibited by the institution is associated with the lack of knowledge of sectoral regulations for the presentation and structuring of medical bills and unification of criteria in these processes. These are the same aspects that has deepened the crisis in the Colombian health sector at the national level.

Keywords: Billing; medical bills; sustainability; state-owned hospital. 


\section{INTRODUCCIÓN}

La profunda crisis por la cual atraviesa el sector de la salud de Colombia se ve agudizada por la complejidad del proceso de cobro de las cuentas médicas de las Instituciones Prestadoras de Servicios de Salud (IPS) a las Empresas Promotoras de Salud (EPS) y a las compañías aseguradoras. En general, muchas IPS presentan problemas en la dispendiosa tarea de presentación de las cuentas, teniendo retrasos en la revisión de la facturación, inconsistencias en la información y devolución de documentación. Lo anterior ha llevado a que en Colombia se glose aproximadamente el 30\% de la facturación, trayendo como consecuencia la escasez de recursos para atender con calidad y dignamente a los pacientes (Revista Semana, 2016). El asunto no es solo la falta de recursos, sino la disponibilidad o flujo de caja de los mismos entre los participantes de la cadena de prestación de servicios.

La crisis financiera por la cual atraviesan las IPS en Colombia se debe en gran medida a que los servicios que prestan han crecido notablemente durante las dos últimas décadas. Sin embargo, los instrumentos de facturación, cobranza y control no han evolucionado al mismo ritmo, presentándose enormes discrepancias en los flujos de información entre los diferentes actores del sistema de salud del país (Ministerio de la Protección Social, 2008). Uno de los factores de mayor incidencia en la crisis financiera de las IPS ha sido el crecimiento de la cartera morosa, debido a que las EPS rechazan un considerable porcentaje de las facturas que reciben, quedando estas glosadas o en reglosa o conciliaciones que en numerosos casos concluyen en cartera morosa y perdidas que deben asumir las IPS (Chavarría, 2018). Adicionalmente, tal como lo señalan Leuro \& Oviedo (2016), entre las problemáticas más recurrentes que desembocan en el aumento de la cartera morosa se encuentra el desconocimiento de la normatividad, la carencia de auditoría previa de cuentas y el desconocimiento de la contratación, entre otros (ver tabla 1).

Tabla 1. Problemáticas más frecuentes del proceso de facturación de cuentas médicas

\begin{tabular}{ll}
\hline & \multicolumn{1}{c}{ Área de Facturación } \\
\hline - & Desgaste general administrativo y operativo por objeciones y altas glosas \\
- & Pérdida de control en cartera y flujo de dinero recaudado \\
- & Carencia de auditoría previa de cuentas \\
- & Nesconocimiento de la contratación \\
- & Debilidad en preparación y divulgación de procesos y procedimientos \\
- & Facturación centralizada \\
- & Re labora contra la glosa \\
- & Paros de personal afectan la producción y los ingresos \\
- & Demoras en información de RIPS \\
- & Auditoría médica de calidad y no de cuentas \\
\hline
\end{tabular}

Fuente: Leuro \& Oviedo (2016).

Diversos estudios han encontrado un aumento progresivo de la cartera de las IPS, según la Superintendencia de Nacional de Salud (2017), la cartera morosa de más de 60 días excede los 3 billones de pesos y entre las razones que en mayor se le atribuye esto se 
encuentran los problemas asociados con los dispendiosos y voluminosos procesos de armado, cobro y pago de cuentas médicas. Adicionalmente, las EPS en la revisión de cuentas suelen encontrar no conformidades parciales o totales, las cuales generan devoluciones y glosas a las IPS, bajo los términos establecidos por la normatividad (Chavarría, 2018; Ministerio de la Protección Social, 2008).

La cartera morosa de las IPS ha crecido desbordadamente durante los últimos años, muy a pesar de los esfuerzos normativos del Ministerio de Salud y Protección Social en cuando a soportes de facturación, causales de glosas y devoluciones, y tiempos para los trámites. Al respecto, la Asociación Colombiana de Hospitales y Clínicas (ACHC) encontró en un estudio realizado a corte de 30 de junio de 2017, que las deudas por los servicios de salud prestados a usuarios del sistema ascienden a 8.2 billones de pesos, siendo el 60\% cartera en mora, razón por la cual los hospitales y clínicas debieron provisionar por deterioro de cartera $\$ 758$ mil millones de pesos (Asociación Colombiana de Hospitales y Clínicas, 2017).

Las dos mayores deudoras en el régimen contributivo son la Nueva EPS y COOMEVA EPS con $\$ 874634$ y $\$ 587309$, respectivamente. En el régimen subsidiado el mayor deudor es Alianza Medellín Antioquia EPS S.A.S. (antes Savia EPS) con \$626893 y Caprecom EPS con $\$ 251047$. La tabla 1 muestra en detalle la deuda total de las EPS, la cartera morosa de 60 y más días y su concentración como porcentaje del total de la deuda. Las cifras dejan ver claramente la problemática con la cual deben lidiar las IPS para cobrar sus facturas por servicios de salud prestados a las EPS y con ello poder contar con recursos. Sin lugar a dudas, esta situación viene afectando el rendimiento económico de las instituciones que prestan servicios de salud y con ello su sostenibilidad y la calidad de los servicios que prestan.

Tabla 2. Concentración de cartera de 60 días y más por las 10 principales deudoras a junio 30 de 2017 (miles de pesos)

\begin{tabular}{|c|c|c|c|c|}
\hline \multirow{2}{*}{ Entidad deudora } & \multirow{2}{*}{60 días y más } & \multirow{2}{*}{ TOTAL } & \multicolumn{2}{|c|}{$\begin{array}{c}\text { Concentración (\%) de } \\
60 \text { días y más }\end{array}$} \\
\hline & & & $\begin{array}{l}\text { Junio } \\
2017\end{array}$ & $\begin{array}{c}\text { Diciembre } \\
2016\end{array}$ \\
\hline 1. NUEVA EPS (ambos regímenes) & 488.446 .951 & 886.660 .258 & $55,1 \%$ & $68,9 \%$ \\
\hline 2. ALIANZA MEDELLIN ANTIOQUIA EPS S. A. S. & 426.771 .961 & 626.893 .501 & $68,1 \%$ & $65,9 \%$ \\
\hline 3. COOMEVA EPS & 363.201 .253 & 587.309 .560 & $61,8 \%$ & $58,6 \%$ \\
\hline 4. CAFESALUD EPS (ambos regímenes) & 301.775 .910 & 486.325 .905 & $62,1 \%$ & $66,7 \%$ \\
\hline 5. SALUDCOOP EPS OC & 285.459.702 & 285.459 .702 & $100,0 \%$ & $100,0 \%$ \\
\hline 6. CAPRECOM & 251.047 .505 & 251.047 .505 & $100,0 \%$ & $100,0 \%$ \\
\hline 7. SALUD VIDA EPS (ambos regímenes) & 162.364 .334 & 196.141 .854 & $82,8 \%$ & $84,4 \%$ \\
\hline 8. OPERADOR FIDUCIDIARIO & 126.652 .471 & 166.327 .276 & $76,1 \%$ & $81,2 \%$ \\
\hline 9. ANTIOQUIA & 115.126 .894 & 147.631 .011 & $78,0 \%$ & $70,5 \%$ \\
\hline 10. COOSALUD & 109.990 .898 & 210.519 .926 & $52,2 \%$ & $54,9 \%$ \\
\hline Total, general & 2.630 .837 .880 & 3.844 .316 .497 & $68,4 \%$ & $72,1 \%$ \\
\hline
\end{tabular}

La región Caribe de Colombia no es ajena a la problemática descrita anteriormente y en particular el departamento de Bolívar. Por ejemplo, en la ciudad de Cartagena, por falta de recursos, se redujo la oferta hospitalaria pública en más de 300 camas, tras los cierres del Hospital Universitario de Cartagena (HUC) y la Clínica Club de Leones en el año 2003 (Viloria, 2005; Arias, Caraballo \& Matos, 2012; Arias, Caraballo \& Muñoz, 2016; Arias, 
Matos y Caraballo, 2016; Arías \& Díaz, 2015; Arias, Caraballo \& Matos, 2015; De La Puente, Correa \& Arias, 2017; De La Puente, Arias, Caraballo \& Matos, 2018; Arias \& Batista, 2018).

En este orden de ideas, resulta importante el estudio de las razones por las cuales se ha profundizado la crisis financiera del sector de la salud a nivel departamental. Por eso, el presente documento tiene como objetivo hace un análisis del proceso de facturación y cuentas médicas de la Empresa Social del Estado (ESE) Hospital Local de Arjona, aplicando instrumentos de captura de información y empleando el diagnóstico organizacional como herramienta de evaluación de la gestión de estos dos procesos, considerados críticos en las organizaciones dedicadas a la prestación de servicios de salud.

La problemática estriba en que con mucha frecuencia se presentan objeciones o "glosas", atribuibles a inconsistencias e irregularidades en los procesos de facturación y presentación de las cuentas médicas. Lo anterior conduce a que no se haga el pago de la cuenta y con ello se desencadene un problema que amenace la sostenibilidad y la calidad de la prestación de los servicios de salud de las organizaciones de este sector.

\section{REVISIÓN DE LITERATURA}

La facturación y cobro de cuentas medicas por venta de servicios de salud es un proceso medular que pretende garantizar la estabilidad y supervivencia del sector salud y de las instituciones prestadoras de servicios de salud, dado que permite el registro, valoración y liquidación de todos y cada uno de los procedimientos, consumos y actividades que conllevan a la atención hospitalaria o ambulatoria de un usuario para hacer efectivo su cobro. Esto implica que las empresas de salud deben trabajar ahora como las demás empresas de producción y venta de servicios, para lo cual se deben crear procesos administrativos que garanticen una gestión efectiva del proceso de la facturación hospitalaria, auditoría médica de cuentas y recuperación de cartera.

Es de anotar que es precisamente en estos procesos administrativos en donde se evidencia las más grandes falencias de las IPS que según Leuro \& Oviedo (2016):

Tienen un común denominador de dicha problemática es la falencia de conocimientos básicos de la normatividad establecida por el estado y de la seguridad social, con referencia a la facturación y la cartera, especialmente en normas de orden público de la misma seguridad social, normas de salud, deficiente conocimiento y aplicación de manuales tarifarios, abusos de la normatividad en contratación y su aplicación adecuada y legal, entre otras causas (p.28).

En este orden de ideas, conviene señalar que el Sistema General Seguridad Social en Salud (SGSSS), con el fin de con fin el mejorar los procedimientos de cobro, verificación y control y pago de los servicios, en el manejo de la información de cuentas médicas y facturación de los servicios de salud prestados estableció un marco normativo basado en lo establecido en la ley 100 de 1993, la Resolución 1479 de mayo de 2015 y Resolución No 5395 de 2013.Adicionalmente, la expedición de la resolución 0837 de junio 23 de 2015 tiene por objeto establecer el procedimiento para el cobro y pago a los prestadores de servicios de salud públicas, privados o mixtos, por los servicios y tecnologías sin cobertura en el Plan Obligatorio de Salud (POS), provistas a los afiliados al Régimen Subsidiado, autorizados por los Comités Técnico Científicos (CTC) u ordenados mediante providencia 
de autoridad judicial. La Ley 100 de 1993 es un importante referente en este trabajo, en lo concerniente a las entidades administradoras de planes de beneficios, quienes a la postre son las directas responsables de la prestación de servicios de salud a una población específica, en razón de un plan de aseguramiento o por disposición del SGSSS. Lo anterior se hace a través del registro individual de prestación de servicios de salud (RIPS). De acuerdo con la Ley 100 de 1993:

El conjunto de datos mínimos y básicos que el Sistema General de Seguridad Social en Salud requiere para los procesos de dirección, regulación y control, y como soporte de la venta de servicio, cuya denominación, estructura y características se ha unificado y estandarizado para todas las entidades a que hace referencia el artículo segundo de la presente ley. (p.65)

Los datos de este registro se refieren a la identificación del prestador del servicio de salud, del usuario que lo recibe, de la prestación del servicio propiamente dicho y del motivo que originó su prestación (diagnóstico y causa externa). En todo este proceso existe un ente público regulador, que en este caso es el Ministerio de la Salud y de Protección Social. Es prudente señalar que la investigación está enmarcada en la normatividad provista por el Sistema General Seguridad Social en Salud, definido por Passos (2015) de la siguiente manera:

Es el mecanismo que se ha establecido para garantizarle a toda la población un mejoramiento de la calidad de vida a través de la prestación de servicios de salud y la implementación de acciones que se desarrollan para ayudar a las personas, familias y comunidades de escasos recursos económicos, en el manejo de los riesgos socioeconómicos y de salubridad. (p. 24)

Es importante resaltar que la IPS Hospital local de Arjona presta sus servicios de conformidad con los parámetros establecidos por el SGSSS, los cuales exigen la realización de auditorías de cuentas con unos parámetros claramente establecidos, partiendo de la descentralización técnica, administrativa y financiera de las entidades de salud, y atendiendo a la implementación de sistemas de facturación y ventas de servicios de salud, aplicando manuales tarifarios y un sistema donde se oferta la prestación o atención en salud a los usuarios o potenciales beneficiarios. Dado lo anterior, resulta conveniente conceptuar la función de la auditoría, la cual según Albrecht (2007):

Es entendida como la evaluación profesional de todas o una parte de las operaciones o actividades de una organización de cualquier naturaleza. Es una verificación formal de los registros, las actividades operativas, las cuentas, el desempeño de una organización y exámenes que concluyen en informes detallados de los hallazgos efectuados por los responsables del análisis (p.79).

Aquí vale la pena aclarar que la auditoria es un examen crítico de todas áreas de gestión de la IPS, pero no académico, que no implica la preexistencia de fallas en la entidad y que persigue el fin de evaluar y mejorar la eficacia y eficiencia de una sección o de un organismo. Cualquier aspecto de la empresa es susceptible a la auditoria y hay tantas formas de ellas como áreas puedan ser objetos de su revisión. El examen efectuado le proporciona al auditor las evidencias para que puedan emitir un informe que resuma su opinión sobre los elementos revisados. Dicho informe constituye, en consecuencia, la conclusión del trabajo de auditoria. 
La evaluación de la gestión administrativa de la IPS Hospital Local de Arjona, respecto al proceso de facturación y cuentas médicas, implica la realización del diagnóstico organizacional, el cual tiene como objetivo realizar un análisis procesal donde se examinan todas las áreas que contempla una empresa en particular para llegar a estudiarlas con profundidad y resolver situaciones que ponen en peligro la estabilidad, sostenibilidad y el buen funcionamiento de la entidad. Según Fleitman (1997) el diagnóstico hace parte de la auditoria, es un componente de esta; para realizar una auditoria completa se debe partir de un diagnóstico general de la organización, es la evaluación integral, es una metodología por medio de la cual se estudian, analizan y evalúan las fuerzas, debilidades, amenazas y oportunidades de la organización.

Es de anotar que el diagnostico en este caso específico, debe estar orientado a identificar las áreas problémicas, relacionadas con los aspectos procedimentales establecidos por el Ministerio de Salud y Protección Social, para el registro, sistematización, valoración de los servicios de salud prestados, como parte de un proceso de planificado de cambios en la gestión administrativa. En este proceso, es necesario conocer la situación actual de la entidad a partir de los resultados e introducir los cambios necesarios para garantizar los ingresos que permitan la supervivencia, crecimiento y desarrollo de la IPS. Para ValdezRivera (1998), el diagnostico organizacional se inscribe dentro de un proceso de gestión preventivo y estratégico. Se constituye como un medio de análisis que permite el cambio de una empresa de un estado de incertidumbre a otro de conocimiento, para su adecuada dirección. Por otro lado, es un proceso de evaluación permanente de la empresa a través de indicadores que permiten medir los signos vitales.

Por su parte, Cummings \& Worley (2001), consideran que el diagnóstico es una herramienta de la dirección y se corresponde con un proceso de colaboración entre los miembros de la organización y el consultor para recabar información pertinente, analizarla e identificar un conjunto de variables que permitan establecer conclusiones.

Con base en los anteriores conceptos se logra comprender el alcance y las técnicas e instrumentos para su realización. Tal como lo afirma Vidal (2004), el diagnóstico organizacional es una herramienta que, a través de instrumentos como preguntas, cuestionarios, entrevistas, permite obtener información sobre diferentes áreas o procesos. Esto contribuye en el proceso de comprensión y evaluación de la organización y con base en ello en la toma de decisiones de mejora. En este caso en particular, el diagnóstico organizacional servirá para analizar los procesos de facturación y cuentas médicas de la ESE Hospital Local de Arjona, con la finalidad de aportar herramientas analíticas para toma de decisiones que mejoren el flujo de caja, rentabilidad y la calidad de los servicios médicos prestados a los pacientes.

\section{METODOLOGIA}

La presente investigación es de tipo descriptiva, porque permite detallar las características que identifican los elementos y componentes de la organización y su interrelación con las auditorías como factor de competitividad (Passos, 2015). Además, accede sin ambages a la determinación de los hechos que conforman el problema de investigación en cuanto a la identificación de formas de conducta y actitudes del personal directamente relacionado con el objeto de estudio en la IPS Hospital local de Arjona. 
El diseño de la investigación es de campo, debido a que los datos se recogieron directamente de la realidad poblacional, por lo que fueron denominados datos primarios; como lo señala Tamayo (1999): "su valor radica en que permiten cerciorarse de las verdaderas condiciones en que se han obtenido los datos, lo cual facilita su revisión posterior o modificaciones en caso de surgir dudas" (p.72). El esquema de campo se apoyó en la categorización del diseño estadístico, ya que facilitó la medición de un conjunto de variables, consistente en el estudio cuantitativo o evaluación numérica de hechos con características similares de manera colectiva. Es conveniente insistir, que los modelos son de estructura metodológica, y no constituyen una camisa de fuerza para el investigador, ya que solo permiten estructurar el diseño investigación propiamente dicho.

El universo sobre el cual se hizo la investigación es la IPS Hospital Local de Arjona. De igual forma, en la presente investigación se seleccionó una población con características similares (empleados con cargos administrativos), a la cual se llamó conjunto de referencia, y sobre ella se hizo todo lo concerniente a la investigación o estudio. Dado lo anterior, el muestreo utilizado fue no probabilístico, esgrimiendo para ello una muestra intencional por conveniencia, constituida por diez (10) empleados administrativos, pertenecientes al área de contabilidad, lo cual permitió realizar un estudio descriptivocorrelacional, donde las auditorías, el capital humano, los procesos de facturación y cuentas médicas, y usuarios son importantes, ya que le permiten a la IPS ser sostenible en un mercado muy competitivo, estructurado y regulado por normas de estricto cumplimiento.

\section{RESULTADOS}

La aplicación del instrumento a los empleados de la IPS Hospital Local de Arjona del área de contabilidad, se realizó con el objetivo de conocer a profundidad la situación actual en relación a los procesos de facturación y cuentas médicas y con ello detectar posibles problemas asociados a inconsistencias que se presentan en las objeciones o glosas, atribuibles a irregularidades en estos procesos; teniendo presente que la IPS cuenta con un amplio portafolio de servicios, clientes y proveedores, los cuales exigen información de calidad, ágil, confiable y oportuna, que hagan posible la liquidación y presentación de facturas a las entidades responsables de los pagos sin errores y contratiempos.

Figura 1. Cuestionario al personal contable en la ESE Hospital Local de Arjona (respuestas a preguntas 1-7)

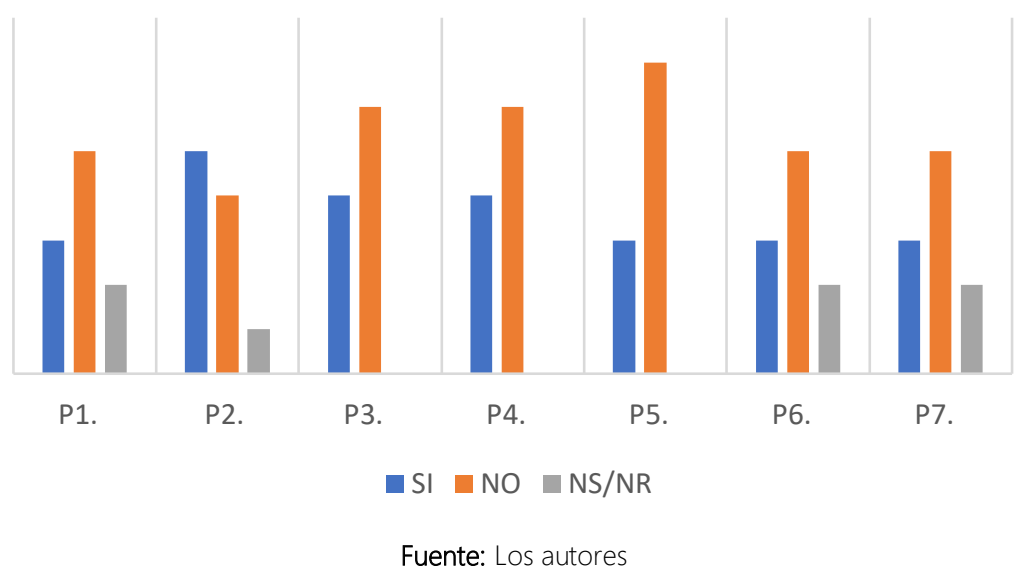


Pregunta 1: ¿En la ESE Hospital Local de Arjona se relaciona la identificación del usuario con el régimen al que pertenece?

Como se observa, 5 de los encuestados (50\%) responde que no existe relación y mucho menos identificación entre el usuario y el régimen al que pertenece, lo que desencadena un caos en el manejo de la información y consecuente con ello en la facturación, desestabilizando lo anterior las débiles finanzas de la empresa. Así mismo, 3 (30\%) afirman que si existe dicha relación y un $20 \%$ no sabe o no responde por desconocimiento.

Pregunta 2: ¿Conoce todos los beneficios establecidos en el POS?

De los encuestados, el 50\% afirma conocer el plan de beneficios a que tiene derecho el usuario que solicita los servicios de la IPS; el 40\% manifiesta no conocerlo y el 10\% no sabe o no responde. Lo anterior indica, que 90\% de los encuestados tiene plena pertinencia y pertenencia con los servicios que ofrece la organización, los que consideran de la más alta calidad y a la altura de los mejores del departamento y la región.

Pregunta 3: ¿Tiene conocimiento de la vigencia de la relación contractual, disponibilidad presupuestal y los tipos de servicio objeto del cobro?

El $40 \%$ de los encuestados tiene conocimiento de la forma como se viene dando la relación contractual, como se ejecuta la disponibilidad presupuestal y los tipos de servicios objeto de cobro que realiza la IPS; mientras que un 60\% (lo cual es medio - alto) desconoce totalmente la relación expuesta. Lo anterior es preocupante, como quiera que, al momento de realizar una auditoría por parte de cualquier ente de control de estado, se le puede dar una información equivocada sobre la cual eleve una glosa y sus respectivas sanciones.

Cabe destacar, que el desconocimiento de los empleados en cuanto a contratación, presupuesto y recaudos por servicios, es un riesgo inminente que se convierte en un punto álgido para cualquier empresa social del estado prestadora de servicios de la salud, ya que impide que se logren las metas trazadas por organización en su líneas estratégicas de acción para una determinada vigencia, colocando en tela de juicio la gestión administrativa de sus directivos y la transparencia con que se manejan sus recursos.

Pregunta 4: ¿Se excluye la atención inicial de urgencias a la cual están obligados todos los prestadores de servicios de salud, sin que medie relación contractual alguna?

La gráfica muestra, que el $60 \%$ de los encuestados es de la percepción que en los informes de gestión ante los entes de control no se excluye la atención inicial de urgencias, ya que existe una contratación de peso que los obliga a prestar ese servicio; mientras que el $40 \%$ asegura que se excluyen de los servicios ofertados y de los informes, muy a pesar que ley 100/93 y sus decretos reglamentarios obligan a las prestadoras de servicios de salud a proporcionarlo de manera eficiente y oportuna.

Pregunta 5: ¿Si un servicio o producto no está registrado, soportado y justificado en la historia clínica, el asegurador lo paga?

El 30\% de los consultados expresaron que siempre se hace evaluación de la correlación del estado clínico del usuario y los diagnósticos con el empleo de medicamentos, procedimientos quirúrgicos, laboratorio clínico y de imágenes diagnósticas, lo mismo que 
distintos recursos diagnósticos y terapéuticos. Por otra parte, el $60 \%$ restante afirma que este control y/o evaluación jamás se hace.

Pregunta 6: ¿Si el estado clínico del usuario no corresponde al diagnóstico que soportó las decisiones del profesional de la salud o con los protocolos internos de la IPS, o el diagnostico no se correlaciona con los recursos utilizados, se considera el uso injustificado de estos, y las EPS los paga?

Es importante resaltar que el 50\% de los encuestados considera que cuando el estado clínico del usuario no corresponde al diagnóstico que soportó las decisiones del profesional de la salud, o en su defecto este diverge de los protocolos internos de la IPS y no se correlaciona con los recursos utilizados, las EPS no los paga; mientras que un 30\% considera el uso injustificado de estos servicios, los cuales son cobrados a las EPS y lógicamente estas lo cancelan formalmente. Cabe destacar que un 20\% no sabe (desconoce) o se abstiene de responder ante la pregunta formulada.

Pregunta 7: ¿Existe un proceso formal para la radicación, auditoria y respuesta de glosas? Entre la población encuestada se encontró, que un 30\% comparte la existencia de un proceso formal para la radicación, auditoría y respuestas de glosas, un 50\% niega rotundamente tal existencia y un $20 \%$ no sabe o no responde. Asimismo, el $90 \%$ de la muestra en cuestión es partidaria que la IPS cuente con una herramienta de apoyo para el proceso de facturación y cuentas médicas, ya que su uso permitiría el levantamiento de glosas por parte de los entes de control del estado.

\section{Gráfico 2. Cuestionario al personal contable en la ESE Hospital Local de Arjona (respuestas a preguntas 8-17)}

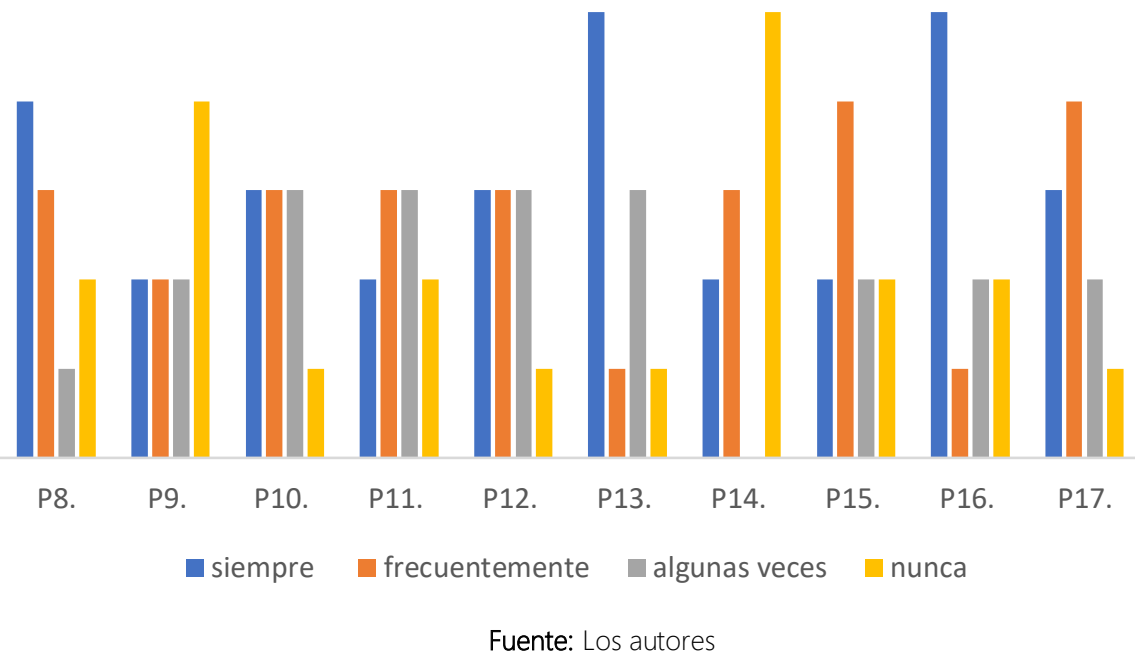

Pregunta 8: ¿Está al tanto que se comprueben los derechos como usuario previa prestación del servicio, o en su defecto cuando éste ya se ha prestado?

El 40\% de los encuestados está al tanto que se comprueben siempre los derechos que tienen los usuarios previa prestación del servicio, o en su defecto cuando éste ya se ha cumplido; el 30\% lo hace frecuentemente, el 10\% lo hace algunas veces y el 20\% nunca está al tanto, ni les interesa los derechos que tienen los usuarios de la IPS. 
Pregunta 9: ¿Cuándo auditoría médica de cuentas (AMC) efectúa la revisión de la facturación, visa lo que el auxiliar de facturación en un primer momento relacionó?

El 60\% de los encuestados (siempre, frecuentemente y algunas veces) afirma que en los períodos de revisión y/o auditoría médica, la AMC visa lo que el auxiliar de facturación en un primero momento relacionó, lo que se convierte en una debilidad para la IPS como quiera que en pre-informes e informes definitivos concurren hallazgos contables y/o financieros que merecen espacial atención por parte de la administración, si se quieren evitar en lo sucesivo glosas u observaciones de parte de los entes de control. Por otro lado, el $40 \%$ de los encuestados manifiesta que nunca la AMC ha visado en un primer momento la facturación.

Pregunta 10: ¿Se comprueba en la ESE Hospital Local de Arjona que los servicios que el usuario recibió fueron los que efectivamente necesitaba de acuerdo al estado clínico en que se encontraba y al diagnóstico que se le efectuó?

El 90\% de los encuestado manifiesta fidedignamente, que en la IPS el usuario recibe los servicios que efectivamente necesitaba de acuerdo a su estado clínico y al diagnóstico que se le efectuó; más, sin embargo, un 10\% expresa que nunca el servicio obedece a la condición clínica que presenta el paciente y mucho menos a los prescrito por el médico de turno. Llama la atención el último hallazgo, pues en múltiples oportunidades las IPS son señaladas de facturar a cualquier precio, incluso por servicios de salud que muchas veces no están en capacidad de prestar.

Pregunta 11: ¿Se hace seguimiento y evaluación desde las auditorías de cuentas médicas a la calidad del diligenciamiento de los registros de atención?

El $60 \%$ de la población objeto de estudio manifiesta, que frecuentemente y/o algunas veces se hace seguimiento y se evalúa desde las auditorias de cuentas médicas la calidad del diligenciamiento de los registros de atención a los usuarios, mientras que un 20\% expresa, que siempre se hace seguimiento a los registros de atención, y el restante 20\% afirma que nunca se ha ejecutado tal valoración a la calidad de los servicios a sus respectivos registros

Pregunta 12: ¿Se hace evaluación de la correlación del estado clínico del usuario y los diagnósticos con el empleo de medicamentos, procedimientos quirúrgicos, laboratorio clínico y de imágenes diagnósticas, lo mismo que otros recursos diagnósticos y terapéuticos?

El 30\% de los entrevistados manifiesta que muchas veces el asegurador cancela los servicios o productos no registrados, soportados y justificados en la historia clínica de sus usuarios; mientras que un $70 \%$ expresa que no.

Pregunta 13: ¿Se verifica que los servicios facturados hayan sido efectivamente prestado y recibidos por el usuario?

El 50\% de lo encuestado manifiesta que siempre se verifica que los servicios facturados hayan sido efectivamente prestado y recibidos por el usuario; el 30\% di afirma que algunas veces, el $10 \%$ señala que se hace frecuentemente la verificación, mientras que el $10 \%$ indica que este control jamás se hace.

Pregunta 14: ¿Se examina con los responsables técnico-científicos y administrativos los costos y consecuencias de la inadecuada facturación sobre las finanzas de la empresa? 
El 20\% de la población objeto de estudio señala que siempre, mientras que el 30\% dice que frecuentemente, y el 50\% expresa que nunca. Lo anterior indica que cuando hay fallas en el control de ingresos y gastos de los establecimientos públicos, así como en la totalidad de sus recursos financieros disponibles y sus bienes, estos serán vigilados de la misma manera como se ejerza la gestión gubernamental y según las reglamentaciones prescritas o que prescriban por los entes de control.

Pregunta 15: ¿se verifica la calidad y la suficiencia de los suministros proporcionados por los prestadores contratados, en cumplimiento de las obligaciones con los usuarios, definidas en la integralidad de la atención y la cobertura del plan obligatorio de salud? Un 40\% de los encuestados cree que frecuentemente se verifica; un 40\% es de la opinión que siempre y/o algunas veces se hace tal cotejo, y un 20\% afirma que nunca se ha dado el referido control, violando de esta manera las resoluciones y acuerdo establecidos y definidos por el Ministerio de Protección Social para los prestadores de salud en Colombia.

Pregunta 16: ¿se verifica que el monto de servicio facturado esté de acuerdo a las tarifas acordadas?

El 50\% de los funcionarios de la IPS afirman que siempre se evidencia, que el monto de los servicios facturados esté de acuerdo a las tarifas convenidas y/o contratadas con las EPS o con aquella empresa prestadora de servicios de salud que los solicite; el 20\% manifiesta que es una labor que se realiza de vez en cuando en la organización, el 10\% justifica que es una actividad que se ejecuta con frecuencia y el $20 \%$ estima que nunca se ha realizado dicha labor. Según este resultado, es necesario establecer criterios bien que permitan una verdadera transparencia en las cuentas que factura la IPS a las EPS o ARSS de la ciudad y la región, entendiendo que para ello debe existir una correlación permanente entre la cantidad de servicios contratados, más la cantidad de servicios prestados y lógicamente los facturados en una determinada vigencia y/o periodo por la empresa. Si existe un control eficiente desde este punto de vista, se evitaría un detrimento de los recursos que por ley son convenidos o contratados, lo que le garantizaría tanto a las EPS, ARSS y ESE un desarrollo sostenible y sustentable en el tiempo.

Pregunta 17: ¿Se verifica la exactitud en el cobro de los afiliados y beneficiarios de las cuotas moderadoras y copagos reglamentados por el CNSSS, cuando contractualmente acuerdan que el prestador sea quien efectúa estos recaudos?

Los encuestados afirman en un $40 \%$ que frecuentemente se verifica la exactitud en el cobro a los afiliados y beneficiarios de las cuotas moderadoras y copagos reglamentados por el CNSSS, muy a pesar que contractualmente acuerdan que el prestador sea quien efectué estos recaudos. De igual manera, un 30\% opina que siempre se realiza dicha verificación, un 20\% dice que se hace algunas veces y un 10\% expresa que nunca se hace. 
Gráfico 3. Respuestas de las preguntas 18 a la 22 del personal contable en la ESE Hospital Local de Arjona

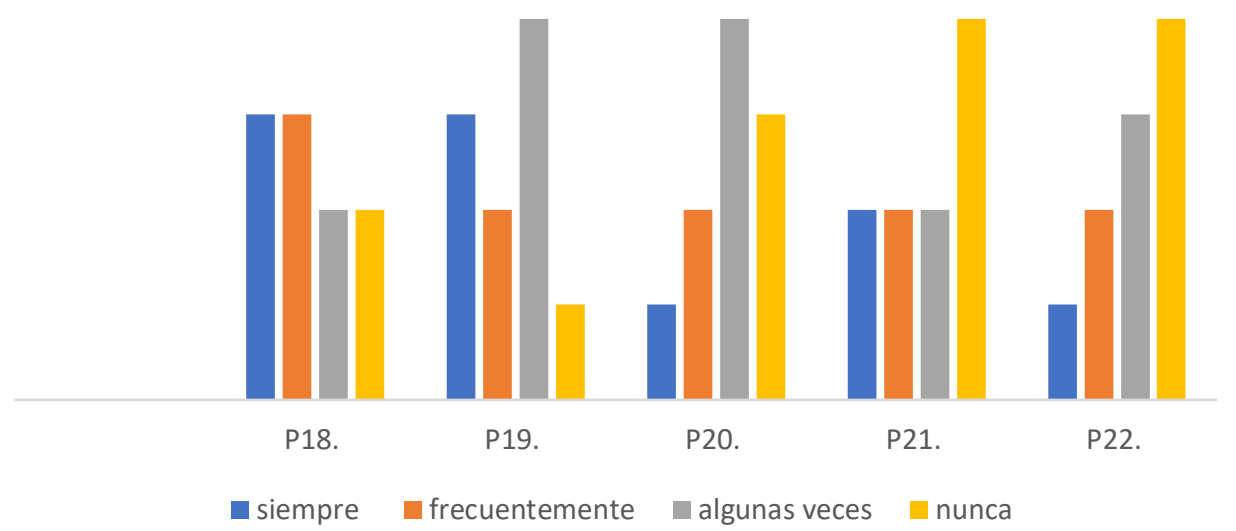

Pregunta 18: ¿Se verifica que las facturas de cobro presentadas por los prestadores de servicios de salud correspondan a las normas tarifarias o a los acuerdos contractuales estipulados?

Es importante señalar que el $60 \%$ de los encuestados expresó que siempre y/o frecuentemente se verifica que las facturas de cobro presentadas por los prestadores de servicios de salud correspondan a las normas tarifarias o a los acuerdos contractuales estipulados; un 20\% refrenda que dicha actividad se realiza algunas veces, y un 20\% afirma que nunca se ha cotejado las facturas con las tarifas convenidas o estipuladas.

Pregunta 19: ¿Se realizan continuamente recomendaciones a la gerencia de la institución para la socialización de las normas, propiedades y atributos de la calidad de los procesos relacionados con contratación, prestación, facturación y cartera?

El 30\% de los encuestados dice que siempre, mientras que el 20\% respondió que frecuentemente, un $40 \%$ opina que algunas veces y el $10 \%$ manifiesta que nunca se ha efectuado sugerencias y/o recomendaciones a la alta dirección de la empresa.

Pregunta 20: ¿Se evalúa y se concreta con las unidades funcionales las normas técnicas, procedimientos y estándares definidos alrededor de los procesos?

En cualquier empresa pública o privada es importante evaluar y concretar con las unidades funcionales, las normas técnicas, procedimientos y estándares definidos alrededor de los procesos de facturación y auditoría; sin embargo, sobre este aspecto, el $30 \%$ de los indagados afirma que dicha evaluación jamás se ha realizado en la IPS, mientras que el $40 \%$ dice que se hace algunas veces, el $20 \%$ que se realiza frecuentemente y el $10 \%$ se pronuncia diciendo que siempre.

Pregunta 21: ¿Se identifican los problemas que motivan las glosas y el no pago de una factura y se asesora el proceso de toma de decisiones para su mejoramiento?

El $40 \%$ de los entrevistados afirman que se identifican los problemas que motivan las glosas y el no pago de una factura, exponiendo detalladamente que muchas veces no se asesora el proceso de toma de decisiones para su mejoramiento; y el 60\% expresa, que siempre, frecuentemente $\mathrm{y} / \mathrm{o}$ algunas veces se identifican los acontecimientos 0 
anormalidades que presentan las cuentas, generando lo anterior hallazgos fiscales y disciplinarios donde se ven involucrados distintos funcionarios.

Pregunta 22: ¿Existe un programa permanente de mejoramiento continuo de los procesos de contratación, prestación, facturación y cartera, con base en los resultados de las actividades de auditoría de cuentas médicas?

En relación a si existe un programa permanente de mejoramiento continuo de los procesos de contratación, prestación, facturación y cartera, con base en los resultados de las actividades de auditoría de cuentas médicas de la IPS, el 40\% de los encuestados dice que nunca se ha realizado; el 30\% expone que algunas veces se realiza un plan de mejora; el $20 \%$ expresa que con frecuencia se ejecutan tales programas y un $10 \%$ afirma que siempre se han efectuado. Lo anterior deja ver que la IPS no realiza un proceso de autoevaluación riguroso, cuya planificación, organización, ejecución y supervisión esté a cargo de personas comprometidas con la misma organización, entendiendo que la autoevaluación persigue dos propósitos centrales: identificar los problemas, dificultades, aciertos y logros de un programa (fortalezas y debilidades), y proponer correctivos y comprometerse en la revisión y ajuste para garantizar un proceso permanente de mejoramiento cualitativo organizacional desde todo punto de vista.

\section{DISCUSIÓN}

La facturación y cuentas medicas son procesos medulares en la ESE Hospital Local de Arjona, en los cuales, a pesar de los esfuerzos realizados por la administración, se presentan debilidades e inconsistencias, generalmente por la falta de control en la facturación, ocasionando re-procesos que implican la revisión y auditoria de los servicios prestados, lo cual conduce a demoras en los tiempos de respuestas a las glosas presentadas por las entidades responsables de pago, lo que por consiguiente afecta el flujo de caja, poniendo en peligro la sostenibilidad económica y financiera de la IPS estudiada. Lo anterior es consistente con las respuestas de las preguntas 21 y 22 y con los resultados de Leuro \& Oviedo (2016) acerca de las problemáticas más frecuentes del proceso de facturación de cuentas médicas.

Cabe señalar que la facturación y las cuentas medicas no son procesos aislados, sino que dependen en gran medida del flujo de información que se genera de desde la caracterización del paciente al ingreso al hospital, para acceder a los diferentes servicios de acuerdo a la patología, esto produce información valiosa que necesariamente deben aparecer en los RIPS (Registro Individual de Prestación de Servicios), los cuales se refieren al conjunto de datos que identifican una a una las actividades de salud que se realizan a las personas y que hacen parte integral de la factura de venta de servicios de salud (Resolución 3374 de 2000). Es de anotar que el marco legal es claro, lo que se debe entrar a revisar es si el personal a cargo de la prestación del servicio efectivamente realiza el registro de la información de acuerdo al tipo de usuario y proveedor. En ese mismo sentido, el desconocimiento de los empleados, hace que incurran en errores, en cuanto a contratación, presupuesto y recaudos por servicios, los que a la postre se transforman en riesgos inminentes que se han convertido en un punto álgido para cualquier ESE prestadora de servicios de salud, puesto que impide que se logren las metas fijadas por organización en su líneas estratégicas de acción para una determinada vigencia, 
reflejando debilidades en la gestión administrativa de sus directivos y la transparencia con que se manejan sus recursos, amenazando la sostenibilidad de la misma.

Diferentes trabajos evidencian la existencia de deficiencias en el proceso de facturación y presentación de cuentas médicas a las instituciones responsables del pago por la prestación de estos servicios de salud (Asociación Colombiana de Hospitales y Clínicas, 2017; Chavarría, 2018; Leuro \& Oviedo, 2016). En ciertos casos esto ocurre por omitir la verificación de las tarifas contractuales. Si bien, los resultados muestran que en la mayoría de casos se ajustan a las tarifas contractuales, resulta necesario revisar permanentemente los criterios y realizar los ajustes necesarios para garantizar transparencia y cumplimiento de la normatividad en las cuentas que factura la IPS a las EPS o ARSS de la ciudad y la región, entendiendo que para ello debe existir una correlación permanente entre la cantidad de servicios contratados, más la cantidad de servicios prestados y lógicamente los facturados en una determinada vigencia y/o periodo por parte de la entidad. Si existe un control eficiente desde estos criterios, entonces se evitaría un detrimento de los recursos que por ley son convenidos o contratados, lo que le garantizaría tanto a las IPS, ARSS y ESE un desarrollo sostenible y sustentable en el tiempo.

La detección de los puntos críticos del proceso es clave en la minimización de los riesgos derivados de errores en la liquidación de las facturas y con ello evitar que las entidades pagadoras (EPS/ARSS) la conviertan el glosa (no pago), desatando con ello todo un proceso de análisis y validación de estas objeciones a la factura, que finalmente se materializa como un reproceso o lastre, afectando a los indicadores, incrementando los costos e impactando negativamente los flujos de ingresos y las finanzas de la ESE Hospital Local de Arjona. Esto implica que se generan problemas de liquidez para el pago de proveedores, empleados, servicios públicos, los cuales afectan el crecimiento, la supervivencia, la sostenibilidad y gestión administrativa de la empresa.

Finalmente, los resultados y la discusión de la presente investigación se orientan a la optimización del proceso de facturación y presentación de cuentas médicas, dada la necesidad de identificar los puntos críticos del proceso y minimizar las glosas y reproceso, las cuales amenazan las sostenibilidad y calidad de los servicios de la ESE Hospital Local de Arjona.

\section{CONCLUSIONES}

A partir de los resultados del estudio de diagnóstico organizacional, aplicado a los procesos de facturación y cuentas médicas en la ESE Hospital Local de Arjona, se concluye que las inconsistencias y deficiencias presentes en estos procesos se atribuyen a irregularidades en la facturación, armado y presentación de dichas cuentas. Lo anterior se debe al desconocimiento de la normatividad para la presentación de las mismas y guarda estrecha relación con las debilidades detectadas en los mecanismos de control y auditoria, con la ausencia de unificación de criterios en estos procesos y la escasa capacidad de respuesta para responder oportunamente a las controversias o desacuerdos, generados en la presentación de las cuentas, ante las entidades encargadas del pago (EPS-ARSS), las cuales glosan el pago y con ello se vea amenazada la sostenibilidad y la prestación de servicios médicos de calidad por parte de la ESE Hospital Local de Arjona. 
Las respuestas de las preguntas 16 a la 22 confirman sendas debilidades operacionales y administrativas en materia de: a) verificación de montos y servicios facturados acorde a las tarifas vigentes; b) exactitud de los cobros, acuerdos contractuales; c) estandarización y unificación de criterios; d) identificación de los motivos del no pago o glosa de las cuentas médicas; e) recomendaciones a la gerencia en los procesos de contratación, prestación, facturación y cartera y f) mejoramiento continuo de los procesos de contratación, prestación, facturación y cartera. En virtud de lo anterior, y dada la amenaza a los ingresos y sostenibilidad financiera de la institución, se recomienda la actualización y capacitación del personal responsable de la facturación y presentación de cuentas médicas en la normatividad vigente inherente a dicho proceso y la implementación de mecanismos de verificación, control, seguimiento, auditoria, retroalimentación de los procesos, respuesta a las glosas y mejora continua, los cuales sean ampliamente conocidos y socializados, logrando con ello la unificación de criterios en materia de estos procesos críticos y vitales para la sostenibilidad de la ESE Hospital Local de Arjona.

\section{REFERENCIAS}

Albrecht, K (2007). Servicio al cliente interno: cómo solucionar la crisis de liderazgo en la gerencia intermedia. España: Ediciones Paidós.

Arias, F \& Batista, A. (2018). La actividad del turismo médico en Cartagena de Indias: Un enfoque del lado de la oferta. Brújulas investigativas de turismo, administración y comercio. Cartagena: Editorial de la Institución Tecnológica Colegio Mayor de Bolívar.

Arias, F, Caraballo, A \& Matos, R. (2016). Turismo médico: caracterización de su oferta en el distrito de Cartagena. Cartagena: Editorial Bonaventuriana.

Arias, F. \& Díaz, K. (2015). El turismo médico en Cartagena de indias: directrices para su desarrollo. Méthodos $15, \quad 80 \quad$ - $95 . \quad$ Recuperado de: http://openjournalsys.colmayorbolivar.edu.co/index.php/Methodos/article/view/16/34

Arias, F. J. A. Matos, R. E. N. \& Caraballo, A. M. P. (2016). Turismo Médico: Caracterización de su Oferta en el Distrito de Cartagena. Cartagena: Editorial Bonaventuriana.

Arias, F.J., Caraballo, Muñoz, J. M. (2016). El turismo médico en Cartagena: "oferta y barreras". Dimensión Empresarial, 14(2), 143-162.

Arias, F.J.; Caraballo, A.M. Matos, R.E. (2015). La oferta de turismo médico en la ciudad de Cartagena. Sotavento MBA, 25, 10-16. Recuperado de: https://revistas.uexternado.edu.co/index.php/sotavento/article/view/4489/5380

Arias-Aragonés, F. J., Caraballo-Payares, A. M., \& Matos-Navas, R. E. (2012). El turismo de salud: conceptualización, historia, desarrollo y estado actual del mercado global. Clío América, 6(11), 72-98.

Asociación Colombiana de Hospitales y Clínicas. (2017). La realidad financiera de los hospitales y clínicas. Disponible en: http://achc.org.co/wp-content/uploads/2018/01/Realidad-FinancieraHospitales.pdf

Chavarría, T. (2018). Desarrollo e implementación de una herramienta para la validación de cuentas médicas y gestión de glosas en el sector salud en Colombia. Medicina U.P.B. 37(2), 131-141.

Cummings, T. y Worley, C. (2001). Organization development and change. South Western College Publishing.

De La Puente, M, Arias, F, Caraballo, A \& Matos, R. (2018). Desplazamiento transnacional con fines sanitarios. Cartagena: Editorial Bonaventuriana.

De La Puente, M., Correa, J. \& Arias, F. (2017). Aproximaciones teóricas y conceptuales al aseguramiento sanitario desde la noción del actor no estatal en el sistema internacional. Méthodos 75, 94-108.

Fleitman, J. (1997). Evaluación Integral. Manual para el Diagnóstico y solución de Problemas de Productividad, Calidad y Competitividad. México: McGraw-Hill.

Leuro, M. \& Oviedo, I. (2016). Facturación y auditoría de cuentas en salud. Ecoe ediciones.

Ley 100 de 1993. (2006) Bogotá D. C.: Editorial Libros \& Libros, p. 37.

Ministerio de la Protección Social. (2008). Resolución 3047. Recuperado de: https://docs.supersalud.gov.co/PortalWeb/Juridica/OtraNormativa/R MPS 3047 2008.pdf 
Passos, E. S. S (2015). Metodología para la presentación de trabajos de investigación. Una manera práctica de aprender a investigar investigando. Cartagena: Alpha Editores

Revista Semana. (2016, 17 de septiembre). La crisis de las EPS que está dejando a miles de colombianos sin servicios de salud. Recuperado de https://www.semana.com/nacion/articulo/crisis-en-las-eps-y-hospitales-afectan-servicios-desalud-de-miles-de-colombianos/494041

Superintendencia Nacional de Salud. (2017). Informe de acompañamiento pre-jornada de conciliación departamentos del Meta, Guaviare, Vichada y Vaupés. Recuperado de: https://docs.supersalud.gov.co/PortalWeb/Controllnterno/InformesEstatutoAnticorrupcion/Aco mp\%20pre-jorn\%20Villavicencio\%203-2017-008266\%2030-05-2017.pdf

Valdez Rivera, S. (1998). Diagnóstico empresarial. Método para identificar, resolver y controlar problemas en las empresas. México, D.F.: Trillas

Vidal, E. (2004). Diagnóstico Organizacional: Evaluación Sistema de Desempeño Empresarial en la era Digital. Bogotá: Ecoe Ediciones.

Viloria, J. (2005). Salud Pública y Situación Hospitalaria en Cartagena. Banco de la República, Documentos de Trabajo Sobre Economía Regional No.65. Disponible en: http://www.banrep.gov.co/sites/default/files/publicaciones/archivos/DTSER-65-\%28VE\%29.pdf 